\title{
Synergistic antitumor effects of tanshinone IIA and sorafenib or its derivative SC-I in hepatocellular carcinoma cells
}

This article was published in the following Dove Press journal:

OncoTargets and Therapy

\author{
Chien-Ming Chiu' \\ Sung-Ying Huang ${ }^{2}$ \\ Shu-Fang Chang ${ }^{3}$ \\ Kuan-Fu Liao ${ }^{4,5}$ \\ Sheng-Chun Chiu ${ }^{3,6,7}$ \\ 'Division of Colorectal Surgery, \\ Taichung Tzu Chi Hospital, Buddhist \\ Tzu Chi Medical Foundation, \\ Taichung, Taiwan; ${ }^{2}$ Department of \\ Ophthalmology, Hsinchu Mackay \\ Memorial Hospital, Hsinchu, Taiwan; \\ ${ }^{3}$ Department of Research, Taichung \\ Tzu Chi Hospital, Buddhist Tzu Chi \\ Medical Foundation, Taichung, Taiwan; \\ ${ }^{4}$ Graduate Institute of Integrated \\ Medicine, China Medical University, \\ Taichung, Taiwan; ${ }^{5}$ Department \\ of Internal Medicine, Taichung \\ Tzu Chi Hospital, Buddhist Tzu \\ Chi Medical Foundation, Taichung, \\ Taiwan; ${ }^{6}$ Department of Laboratory \\ Medicine, Taichung Tzu Chi Hospital, \\ Buddhist Tzu Chi Medical Foundation, \\ Taichung, Taiwan; ${ }^{7}$ General Education \\ Center, Tzu Chi University of Science \\ and Technology, Hualien, Taiwan
}

Correspondence: Sheng-Chun Chiu Department of Research, Taichung Tzu Chi Hospital, Buddhist Tzu Chi Medical Foundation, 88, Section I, Fengxing Road, Tanzi District, Taichung 42743, Taiwan Tel +8864360 60666 ext 3430 Fax +886 425385958

Email honeyhopes@gmail.com

\begin{abstract}
Introduction: Hepatocellular carcinoma (HCC) is the most common form of hepatic malignancy in the world. We aimed to determine the effect of tanshinone IIA (Tan-IIA) in combination with sorafenib or its derivative SC-1 on cytotoxicity, apoptosis, and metastasis in human HCC cells.
\end{abstract}

Materials and methods: Cytotoxicity was detected by MTT assay. Apoptosis and sub-G1 populations were analyzed by flow cytometry. Cell migration and invasion were evaluated by Transwell assay. Protein expression was detected by Western blot.

Results: Tan-IIA combined with sorafenib or SC-1 exerted synergistic cytotoxicity in HCC cells. Elevated proportions of sub-G1 and caspase activation were observed in the combinative treatments; in addition, marked inhibition of cell migration and invasion, which could be mediated by the modulation of epithelial-mesenchymal transition was observed. pSTAT3 levels were significantly reduced as well.

Conclusion: A combination therapy using Tan-IIA and sorafenib or SC-1 could be a promising approach to target HCC, and further preclinical investigations are warranted to establish their synergetic advantage.

Keywords: tanshinone IIA, hepatocellular carcinoma, sorafenib, SC-1, STAT3, metastasis, combination therapy

\section{Introduction}

Hepatocellular carcinomas (HCCs) are the sixth most common cause of cancer-related deaths among men, with the highest incidence rates in Asia and Africa as reported in 2012., ${ }^{1,2}$ Despite the current advancements in HCC treatment including targeted chemotherapies, survival outcomes for HCC patients are poor, and so more effective therapeutic agents are urgently needed.

Sorafenib, a multi-kinase inhibitor, was the first drug approved for advanced HCC treatment that improves overall survival in HCC patients. ${ }^{3,4} \mathrm{SC}-1$ is a derivative of sorafenib that lacks kinase-inhibition activity while still retaining pSTAT3-repressing activity. ${ }^{5}$ SC-1 was shown to induce apoptosis through SHP-1-dependent STAT3 inactivation and exhibits more potent apoptotic effects than sorafenib does in breast cancer and HCC cells.${ }^{6,7}$ A recent study indicated that HCC patients when treated with a higher dose of sorafenib $(800 \mathrm{mg} / \mathrm{d})$ suffered from more severe side effects, particularly in elderly patients. ${ }^{8}$ Thus, an alternative approach or combination therapies using sorafenib need to be explored for providing improved efficacy without any associated side effects.

Tanshinone IIA $\left(\mathrm{C}_{19} \mathrm{H}_{18} \mathrm{O}_{3}\right.$, Tan-IIA $)$ is a lipophilic compound from a traditional Chinese medicine, Danshan (Salvia miltiorrhiza Bunge), that exhibits antitumor 
activity against various cancer cell lines. ${ }^{9-13}$ Recently, Tan-IIA exerted a cytotoxic effect through apoptosis induction and cell cycle arrest on HCC cells. ${ }^{14-16}$

STAT3, a transcription factor that modulates many genes related to apoptosis and epithelial-mesenchymal transition (EMT), was constitutively activated in many tumors including HCC. ${ }^{17,18}$ Recent reports indicated that pSTAT3 might be increased after chronic exposure to sorafenib treatment in HCC cells, suggesting that inhibition of STAT3 signals is crucial for HCC treatment. ${ }^{19,20}$ Interestingly, our recent study indicated that Tan-IIA suppressed bladder cancer metastasis by modulating pSTAT3 signals. ${ }^{21}$ Therefore, in the present study, we aimed to investigate the combinative synergism of Tan-IIA with sorafenib on HCC treatment.

\section{Materials and methods}

\section{Chemicals and antibodies}

Sorafenib, SC-1, Tanshinone IIA $\left(\mathrm{C}_{19} \mathrm{H}_{18} \mathrm{O}_{3},>97 \%\right.$ HPLC), sodium vanadate $\left(\mathrm{Na}_{3} \mathrm{VO}_{4}\right)$, DMSO, MTT, Tween 20, methanol, and horseradish peroxidase-conjugated secondary antibodies were purchased from Sigma Chemical Co. (St Louis, MO, USA). The antibodies against p-STAT3 (Tyr705), STAT3, CCL2, E-cadherin, N-cadherin, Vimentin, Slug, Snail, and $\beta$-actin were obtained from Cell Signaling Technology, Inc. (Danvers, MA, USA). PVDF membranes, BSA protein assay kit, and Western blot chemiluminescence reagent were purchased from Amersham Biosciences (Arlington Heights, IL, USA).

\section{Cell culture}

The human HCC cell lines Huh7 and HepG2 were purchased from Bioresource Collection and Research Center (BCRC, Hsinchu, Taiwan). Each cell line was cultured as per standard as recommendations by the BCRC. Culture medium, fetal bovine serum, penicillin, and streptomycin were purchased from Invitrogen (Carlsbad, CA, USA). Cell lines were authenticated annually by short-tandem repeat analysis and routinely tested for mycoplasma contamination (BCRC).

\section{MTT assay and synergism evaluation}

Cell viability following treatment with various compounds was evaluated using MTT assay, performed in triplicates. Briefly, cells $\left(4 \times 10^{4} /\right.$ well $)$ were incubated in a 24 -well plate containing $0.5 \mathrm{~mL}$ of serum-containing medium. Cells were allowed to adhere for 18-24 h and washed with PBS. Solutions were always prepared fresh in culture medium by dissolving $0.2 \%$ DMSO (control) or $1.5 \mu \mathrm{g} / \mathrm{mL}$ Tan-IIA in the presence or absence of 2.5 or $5 \mu \mathrm{M}$ sorafenib or $5 \mu \mathrm{M} \mathrm{SC}-1$ before their addition to cells. The drug-containing medium was removed after treatment for $48 \mathrm{~h}$, cells were washed with PBS, and culture medium containing $300 \mu \mathrm{g} / \mathrm{mL}$ MTT was added for $1 \mathrm{~h}$ at $37^{\circ} \mathrm{C}$. After the MTT medium was removed, $0.5 \mathrm{~mL}$ of DMSO was added to each well. Absorbance at $570 \mathrm{~nm}$ was detected using a multi-well plate reader, Infinite 200 Pro Tecan ${ }^{\mathrm{TM}}$ (Tecan, Mannedorf, Zurich, Switzerland). The absorbance of DMSO-treated cells was considered as $100 \%$. The combination index (CI) value was determined according to the Chou-Talalay method using CompuSyn software (ComboSyn, Inc., Paramus, NJ, USA), where a CI value below 1 indicated synergism. ${ }^{22}$

\section{Flow cytometric analysis}

The cell cycle was determined by flow cytometry following DNA staining to reveal the total amount of DNA. Approximately $3 \times 10^{5}$ cells were incubated with or without $1.5 \mu \mathrm{g} / \mathrm{mL}$ Tan-IIA in the presence or absence of 2.5 or $5 \mu \mathrm{M}$ sorafenib or $5 \mu \mathrm{M} \mathrm{SC}-1$ for $24 \mathrm{~h}$ (apoptosis) or $48 \mathrm{~h}$ (sub-G1). Sub-G1 cells were examined by harvesting with trypsin/EDTA, collected, washed with PBS, fixed with cold $100 \%$ ethanol overnight, and then stained with a solution containing $20 \mu \mathrm{g} / \mathrm{mL}$ PI, $0.2 \mathrm{mg} / \mathrm{mL}$ RNase A, and $0.1 \%$ Triton X-100 for $30 \mathrm{~min}$ in the dark. The cells were then passed through an Accuri C6 flow cytometer to measure the DNA content. The data were obtained and analyzed with CFlow ${ }^{\circledR}$ software. Apoptotic cell death was examined using annexin V-FITC detection kits according to the manufacturer's instructions (BD Biosciences, San Diego, CA, USA). Ten thousand events were acquired for each sample and analyzed by the BD Accuri C6 flow cytometer with CFlow software.

\section{Statistical analysis}

All data are shown as mean \pm SD. Statistical differences were analyzed using the Student's $t$-test for normally distributed values.

Western blot, cell migration, and invasion assays were performed as previously described. ${ }^{21}$

\section{Results}

\section{Synergistic effects of Tan-IIA combined with sorafenib or SC-I on cytotoxicity in HCC cell lines}

To investigate the effects of sorafenib and SC-1 on the HCC cell lines, Huh7 and HepG2 cells were treated with increasing doses of sorafenib or SC-1 for $48 \mathrm{~h}$ (Figure 1A). 
A

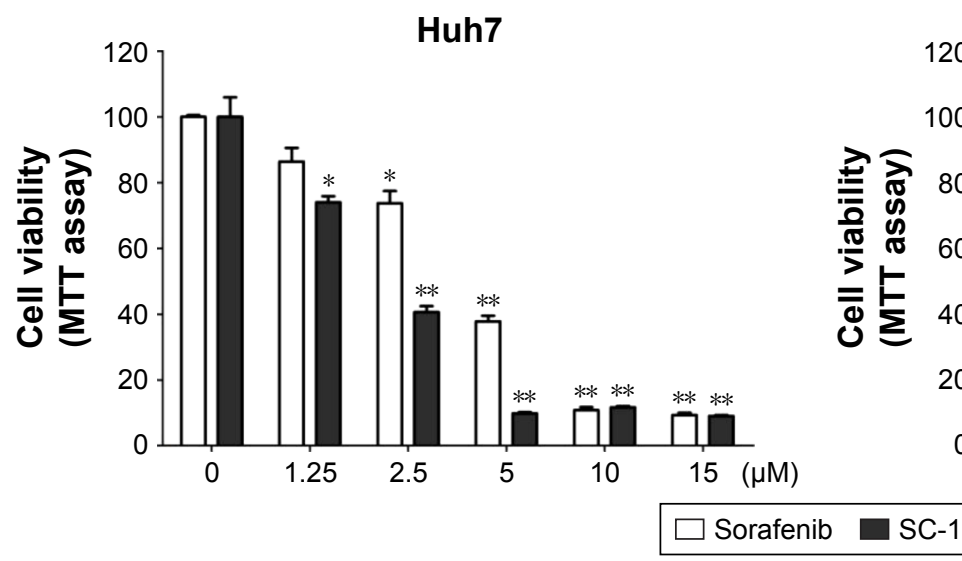

HepG2

B

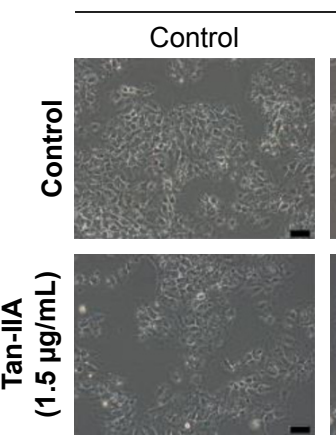

C

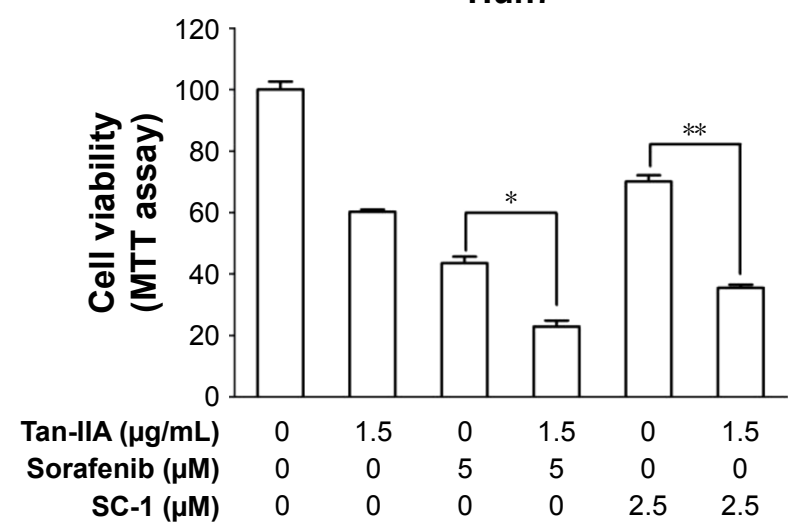

Huh7
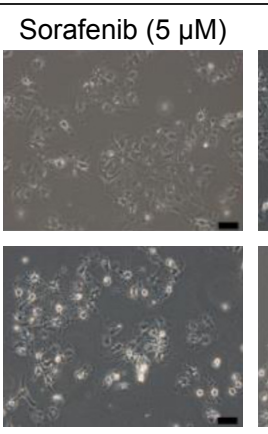

Huh7

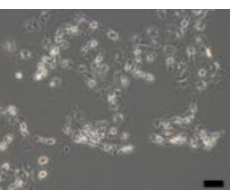

\section{.}

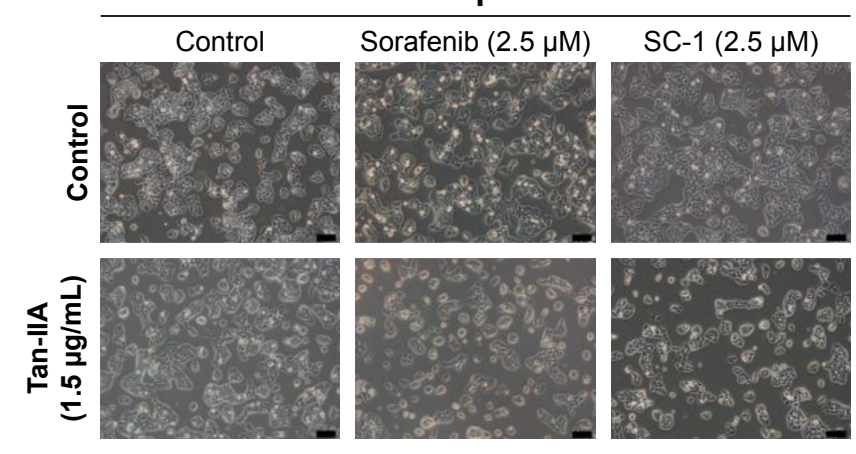

HepG2

HepG2

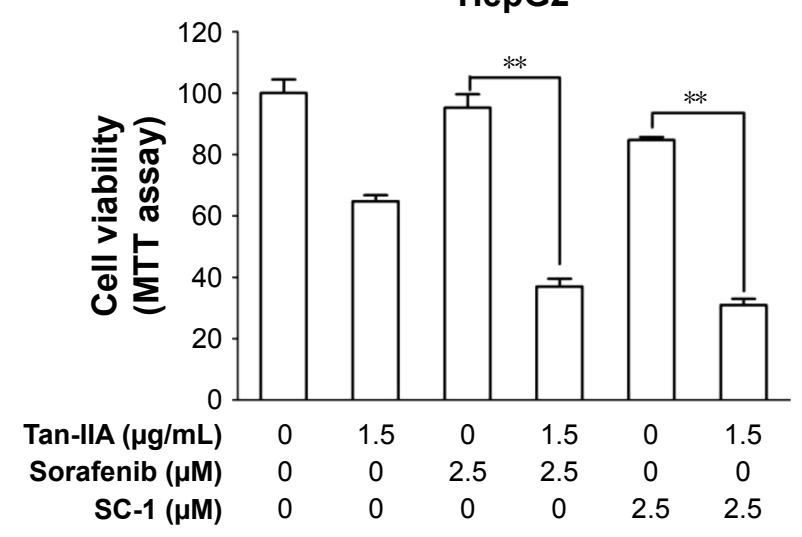

Figure I Effect of cytotoxicity after combination treatment with Tan-IIA and sorafenib/SC-I on HCC cell lines.

Notes: (A) Huh7 and HepG2 were treated with increasing concentrations of sorafenib or SC-I for $48 \mathrm{~h}$ and analyzed by MTT assay. (B) Huh7 and HepG2 cells were treated with or without $1.5 \mu \mathrm{g} / \mathrm{mL}$ Tan-IIA in the presence or absence of 2.5 or $5 \mu \mathrm{M}$ sorafenib, or $2.5 \mu \mathrm{M}$ SC-I for $48 \mathrm{~h}$ followed by MTT assay. (C) Scale bar: $50 \mu \mathrm{m}$. Data are presented as mean $\pm S D$. $*_{p}<0.05$, and $*_{p}^{*}<0.0$ I versus sorafenib/SC-I alone group.

Abbreviations: HCC, hepatocellular carcinoma; Tan-IIA, tanshinone IIA.

The $\mathrm{IC}_{50}$ value for sorafenib was estimated to be $4.5 \mu \mathrm{M}$ (Huh7) and $10 \mu \mathrm{M}$ (HepG2), and for SC-1 was $3.2 \mu \mathrm{M}$ (Huh7) and $4 \mu \mathrm{M}$ (HepG2). To further test the synergistic effects of Tan-IIA combined with sorafenib or SC-1, cells were treated with or without $1.5 \mu \mathrm{g} / \mathrm{mL}$ Tan-IIA in the presence or absence of 2.5 or $5 \mu \mathrm{M}$ sorafenib or $5 \mu \mathrm{M} \mathrm{SC}-1$ for $48 \mathrm{~h}$, then followed by MTT assay (Figure 1B). As shown in Figure $1 \mathrm{C}$, the combination treatment of Tan-IIA with sorafenib suppressed the viability of Huh7 $(22.9 \% \pm 1.9 \%$, CI: 1.07) and HepG2 (37.0\% $\pm 2.5 \%, \mathrm{CI}: 0.41)$ cells compared to the Tan-IIA or sorafenib alone groups. Furthermore, the combination of Tan-IIA and SC-1 suppressed the viability of Huh7 (35.5\% $\pm 1.1 \%$, CI: 0.83) and HepG2 (30.9\% $2.0 \%$, CI: 0.75 ) cells compared to the Tan-IIA or SC-1 alone groups. The CI and dose reduction index values as calculated by the CompuSyn software suggested that these combination 
Table I Cl and DRI of Tan-IIA and Sorafenib/SC-I combination in Huh7 and HepG2 cells

\begin{tabular}{|c|c|c|c|c|c|}
\hline \multicolumn{2}{|c|}{ Concentration } & \multicolumn{2}{|c|}{ Huh-7 } & \multicolumn{2}{|l|}{ DRI } \\
\hline $\begin{array}{l}\text { Tan-IIA } \\
(\mu \mathrm{g} / \mathrm{mL})\end{array}$ & $\begin{array}{l}\text { Sorafenib } \\
(\mu M)\end{array}$ & fa & Cl & Tan-IIA & Sorafenib \\
\hline 1.5 & 1.25 & 0.45 & 1.73 & 0.72 & 2.9 \\
\hline 1.5 & 2.5 & 0.53 & 1.86 & 0.68 & 2.53 \\
\hline 1.5 & 5 & 0.77 & 1.07 & 1.24 & 3.53 \\
\hline $\begin{array}{l}\text { Tan-IIA } \\
(\mu \mathrm{g} / \mathrm{mL})\end{array}$ & SC-I $(\mu \mathrm{M})$ & $\mathrm{fa}$ & $\mathrm{Cl}$ & Tan-IIA & SC-I \\
\hline 1.5 & 1.25 & 0.46 & 0.99 & 1.93 & 2.1 \\
\hline 1.5 & 2.5 & 0.65 & 0.83 & 1.73 & 3.87 \\
\hline 1.5 & 5 & 0.91 & 0.59 & 1.86 & 18.2 \\
\hline \multicolumn{2}{|c|}{ Concentration } & \multicolumn{2}{|c|}{ HepG2 } & \multicolumn{2}{|l|}{ DRI } \\
\hline $\begin{array}{l}\text { Tan-IIA } \\
(\mu \mathrm{g} / \mathrm{mL})\end{array}$ & $\begin{array}{l}\text { Sorafenib } \\
(\mu M)\end{array}$ & fa & $\mathrm{Cl}$ & Tan-IIA & Sorafenib \\
\hline 1.5 & 1.25 & 0.24 & 0.77 & 1.64 & 6.23 \\
\hline 1.5 & 2.5 & 0.62 & 0.41 & 2.88 & 16.54 \\
\hline 1.5 & 5 & 0.78 & 0.54 & 2.16 & $13.8 \mid$ \\
\hline $\begin{array}{l}\text { Tan-IIA } \\
(\mu g / m L)\end{array}$ & SC-I $(\mu \mathrm{M})$ & $\mathrm{fa}$ & $\mathrm{Cl}$ & Tan-IIA & SC-I \\
\hline 1.5 & 1.25 & 0.3 & 0.84 & 1.91 & 3.2 \\
\hline 1.5 & 2.5 & 0.69 & 0.75 & 2.36 & 3.1 \\
\hline 1.5 & 5 & 0.87 & 0.68 & 3 & 2.89 \\
\hline
\end{tabular}

Abbreviations: $\mathrm{Cl}$, combination index; DRI, dose reduction index; fa, fraction affected; Tan-IIA, tanshinone IIA.

treatments exhibited a synergistic effect $(\mathrm{CI}<1)$ on HCC cells (Table 1). In addition, the dose reduction index $>1$ allows dose reduction that leads to toxicity reduction in the therapeutic applications.

\section{Enhanced apoptosis by Tan-IIA in combination with sorafenib or SC-I in HCC cell lines}

To evaluate the role of apoptosis induction by these combination treatments, cells were treated with or without $1.5 \mu \mathrm{g} / \mathrm{mL}$ Tan-IIA in the presence or absence of 2.5 or $5 \mu \mathrm{M}$ sorafenib or $5 \mu \mathrm{M} \mathrm{SC}-1$ for $24 \mathrm{~h}$ (annexin V-FITC) or $48 \mathrm{~h}$ (sub-G1) followed by flow cytometric analysis (Figure 2A and B). The early-apoptotic population (annexin positive/PI negative) was increased by Tan-IIA combined with sorafenib (Huh7: $[9.8 \% \pm 2.4 \%]$ and $\mathrm{HepG} 2:[2.9 \% \pm 0.5 \%]$ cells) and by Tan-IIA combined with SC-1 (Huh7: [6.4\% $\pm 0.4 \%$ ] and HepG2: [2.8\% $0.1 \%]$ cells) (Figure $2 \mathrm{~A}$ ). The sub-G1 population was increased by the combination of Tan-IIA and sorafenib in Huh7 $(25.8 \% \pm 3.9 \%)$ and HepG2 (6.5\% $\pm 2.4 \%)$ cells, and by the combination of Tan-IIA and SC-1 in Huh7 $(8.7 \% \pm 0.4 \%)$ and HepG2 $(14.2 \% \pm 2.9 \%)$ cells (Figure $2 \mathrm{~B}$ ). Activation of caspase proteins is a crucial step for apoptosis induction. We thus examined the protein expression profiles after these test treatments on HCC cells. The expression levels of cleaved caspase- 8 and -3 , and PARP increased after combination treatments with Tan-IIA as compared to that in sorafenib/SC-1 alone group (Figure 2C). Together, these results suggest that these combination treatments can significantly increase the induction of mitochondria extrinsic apoptosis in HCC cells.

\section{Inhibition of migration and invasion by the combination of Tan-IIA treatment and sorafenib or SC-I in HCC cells}

Cancer progression is associated with loss of cell-cell contact and gain of migratory and invasive ability. We therefore assessed the inhibitory effects of these combination treatments on the migration and invasion of cells. Huh7 and HepG2 cells were treated with or without $1.5 \mu \mathrm{g} / \mathrm{mL}$ Tan-IIA in the presence or absence of 2.5 or $5 \mu \mathrm{M}$ sorafenib or $5 \mu \mathrm{M} \mathrm{SC}-1$ for $24 \mathrm{~h}$ (migration) or $48 \mathrm{~h}$ (invasion) and then subjected to Transwell assay (Figure 3A and B). Tan-IIA combined with sorafenib decreased Huh7 (21.3\% $\pm 1.3 \%)$ and HepG2 (116\% $\pm 13.7 \%)$ cell migration, and Tan-IIA combined with SC-1 also decreased Huh7 (6.1\% $\pm 0.7 \%)$ and HepG2 $(24 \% \pm 4.6 \%)$ cell migration as compared to the control group (Figure 2A). Tan-IIA combined with sorafenib decreased Huh7 (27.1\% $\pm 2.5 \%)$ and HepG2 (19.7\% $\pm 2.5 \%)$ cell invasion, and Tan-IIA combined with SC-1 decreased Huh7 $(34.3 \% \pm 4.4 \%)$ and HepG2 $(12.9 \% \pm 4.4 \%)$ cell invasion as compared to the control group (Figure 2B). It has been shown that EMT plays a pivotal role in HCC progression. Thus, the expression profile of EMT-regulatory proteins was also examined after the combination treatments by Western blot (Figure 3C). These combination treatments upregulated the epithelial marker E-cadherin, downregulated the mesenchymal markers N-cadherin and Vimentin, and downregulated the transcription factor Snail and Slug, compared to the sorafenib/SC-1 alone groups. Together, these results suggest that the combination treatments inhibited the migration and invasion of HCC cells, which in turn could be mediated by the modulation of EMT.

\section{Tan-IIA combination treatment with sorafenib or SC-I inhibited proliferation by altering the STAT3 signals in HCC cells} Previous studies have indicated that STAT3 signaling pathway plays a key role in the development of sorafenib resistance in HCC cells. ${ }^{19,23-25}$ To elucidate whether our test combination therapies could alter the STAT3 signals, HCC 


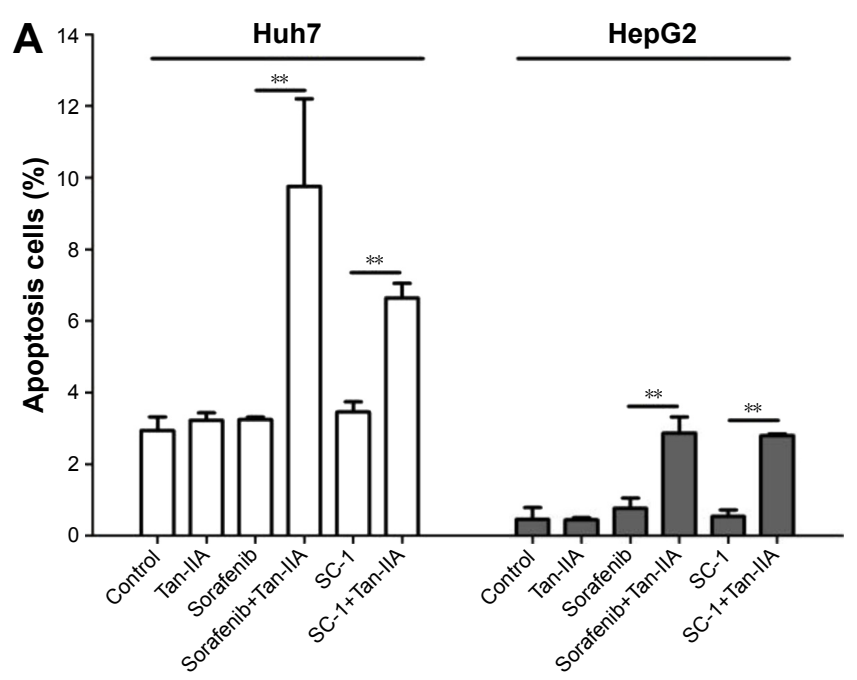

B

Huh7
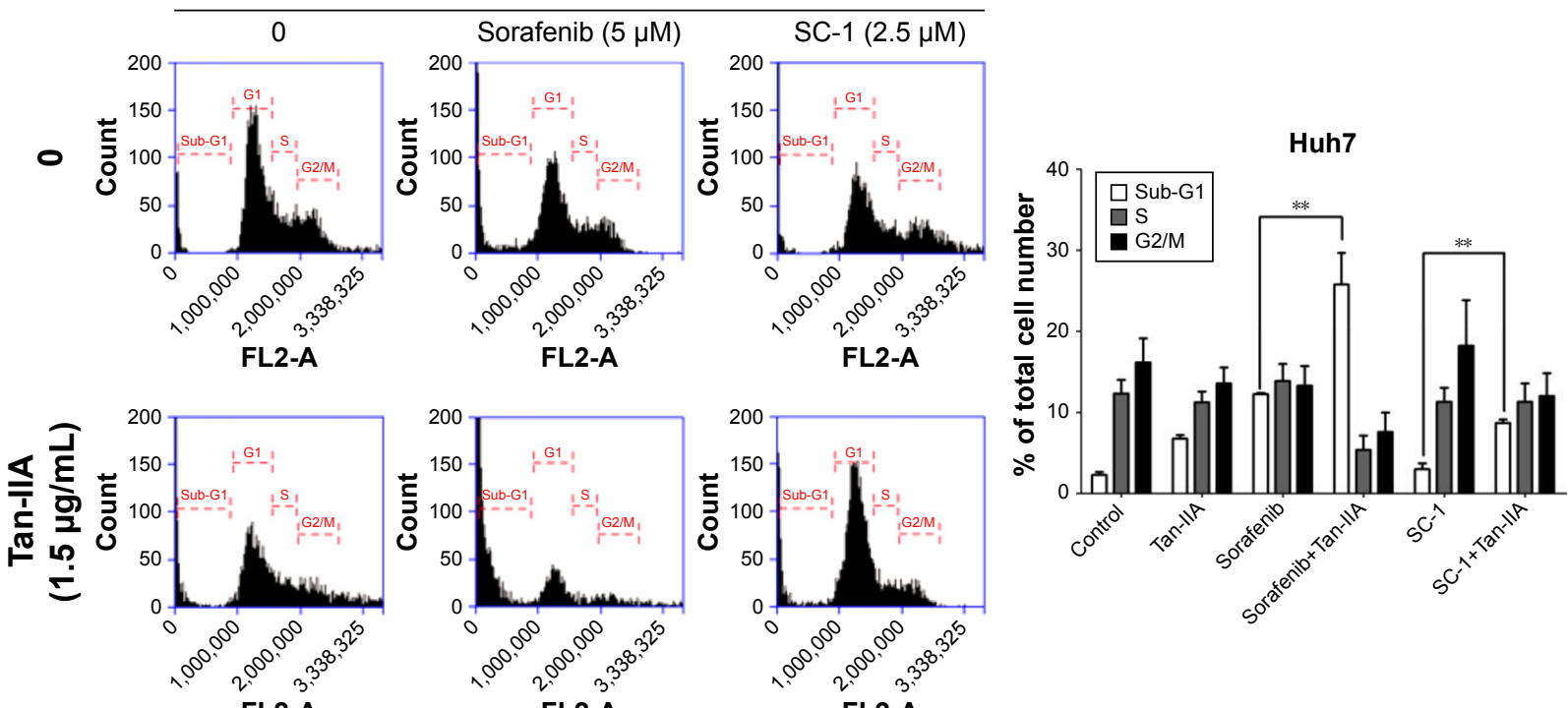

FL2-A

FL2-A

FL2-A

HepG2
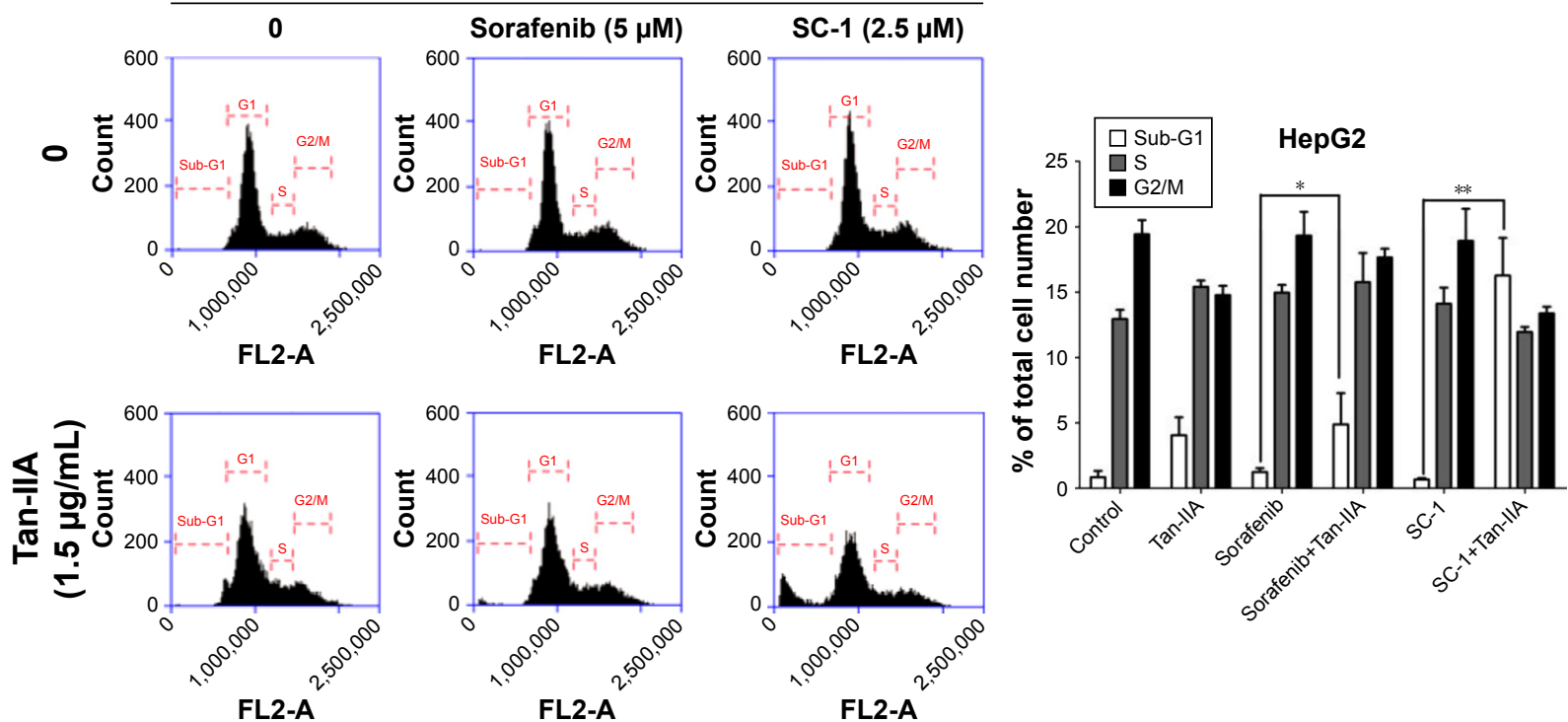

Figure 2 (Continued) 

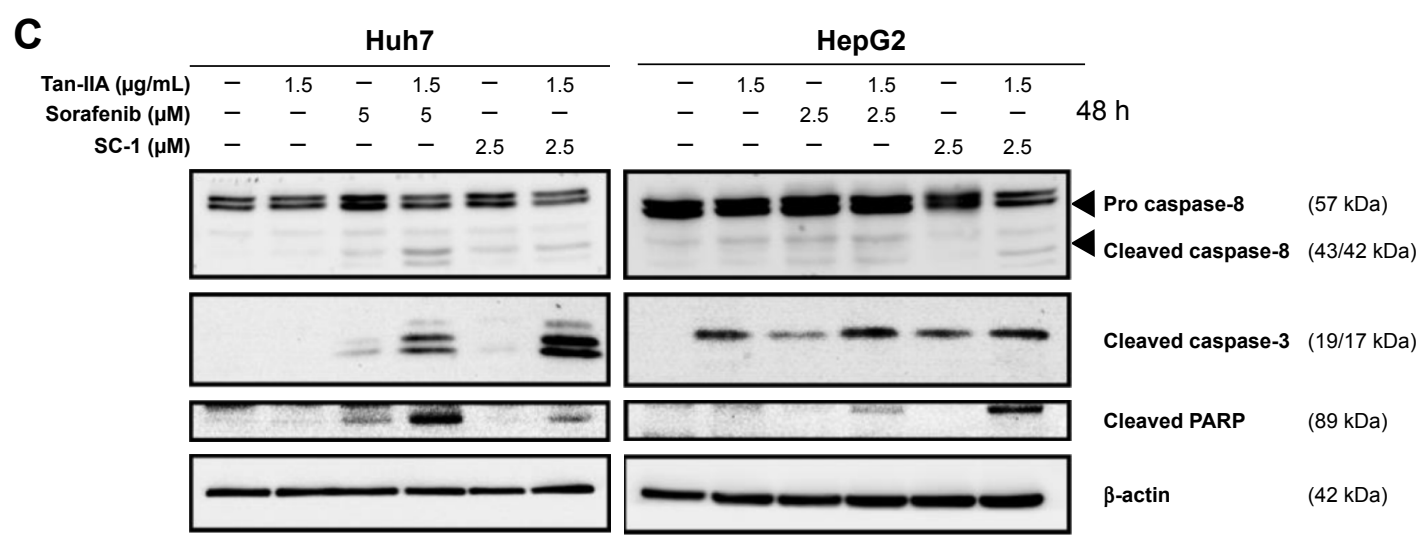

Figure 2 Effect of apoptosis induction after combination treatments with Tan-IIA and sorafenib/SC-I on HCC cell lines.

Notes: Huh7 and HepG2 cells were treated with or without $1.5 \mu \mathrm{g} / \mathrm{mL}$ Tan-IIA in the presence or absence of 2.5 or $5 \mu \mathrm{M}$ sorafenib or $5 \mu \mathrm{M}$ SC-I for $24 \mathrm{~h}$ (A, annexin V-FITC staining) or $48 \mathrm{~h}$ (B, sub-GI analysis) (C, Western blot analysis). Data are presented as mean $\pm \mathrm{SD}$. ${ }^{*} p<0.05$, and ${ }^{* *} p<0.0 \mathrm{I}$ versus sorafenib/SC-I alone group. Abbreviations: HCC, hepatocellular carcinoma; Tan-IIA, tanshinone IIA.

cells were treated with or without $1.5 \mu \mathrm{g} / \mathrm{mL}$ Tan-IIA in the presence or absence of 2.5 or $5 \mu \mathrm{M}$ sorafenib or $5 \mu \mathrm{M}$ $\mathrm{SC}-1$ for $48 \mathrm{~h}$ and then subjected to Western blot analysis (Figure 4A). We observed that these combination treatments greatly suppressed STAT3 signals by reducing p-STAT3 (Y705) protein expression compared to the sorafenib/SC-1 alone group. To test whether the suppression of STAT3 activation is required for the synergistic effects of Tan-IIA, we used the broad-spectrum phosphatase inhibitor sodium vanadate to interfere with STAT3 dephosphorylation. Cotreatment of sodium vanadate with combination treatments restored the phosphorylation of STAT3, leading to a partial rescue of cell death compared to the combination alone group (Figure 4B and C). Thus, the findings indicate that the combination treatments suppressed the growth of HCC cells via suppression of STAT3 signal.

\section{Discussion}

Although sorafenib as a monotherapy has been shown to prolong survival in HCC patients, its adverse effects (such as gastrointestinal bleeding, rash, and hand-foot syndrome) limit its usage under high doses. In addition, there is evidence to suggest that combining sorafenib with uracil-tegafur or octreotide can reduce the dosage of sorafenib, and thus its side effects. ${ }^{26,27}$ Therefore, development of new combination therapies using sorafenib along with other alternative drugs is feasible and promising. A recent study reported that synergistic combination of Tan-IIA and Trans-resveratrol was as effective as cisplatin in the inhibition of HepG2 cell survival, making Tan-IIA a potential candidate for combination treatment for $\mathrm{HCC} .{ }^{28}$ In this study, we demonstrated that synergistic combination of Tan-IIA and sorafenib or
SC-1 enhanced the inhibition on HCC cell growth. These combination treatments enhance the effects of sub-G1 population accumulation and apoptosis induction as well as increase the levels of cleaved caspase- 8 and -3 , and PARP in HCC cells.

There is a growing body of evidence to indicate that the EMT plays a crucial role in the malignant progression of HCC. Our current study demonstrates that combining Tan-IIA with sorafenib/SC-1 caused more potent inhibition of cell migration and invasion than either agent alone. Upregulation of the epithelial marker E-cadherin, downregulation of the mesenchymal markers $\mathrm{N}$-cadherin and vimentin, and downregulation the transcription factors Snail and Slug were observed in HCC cells in response to these combination treatments.

Both sorafenib and SC-1 suppress HCC by pSTAT3 inhibition via the SHP-1-dependent pathway. ${ }^{29}$ However, recent studies indicate that pSTAT3 might be elevated after chronic exposure to sorafenib in HCC cells, thereby suggesting that deregulation of pSTAT3 signals might be linked to sorafenib resistance in HCC treatment. ${ }^{19,23,24}$ Since our recent studies found that Tan-IIA could suppress bladder cancer metastasis by inhibiting pSTAT3 expression, ${ }^{21}$ we combined sorafenib or SC-1 with Tan-IIA to examine their potential synergistic effect to suppress HCC. Our results show that Tan-IIA can suppress HCC proliferation via downregulation of sorafenib/ SC-1-induced pSTAT3, which can be blocked by vanadate treatment.

\section{Conclusion}

Our results suggest that newer combination treatments with Tan-IIA and sorafenib/SC-1 can significantly enhance 
A
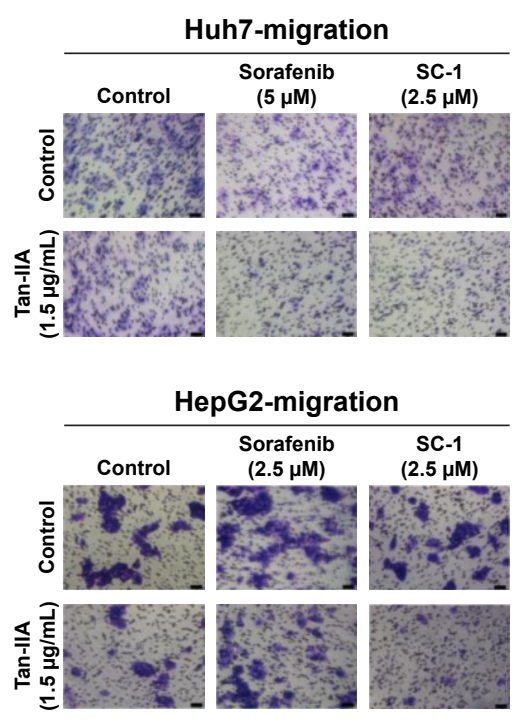

B
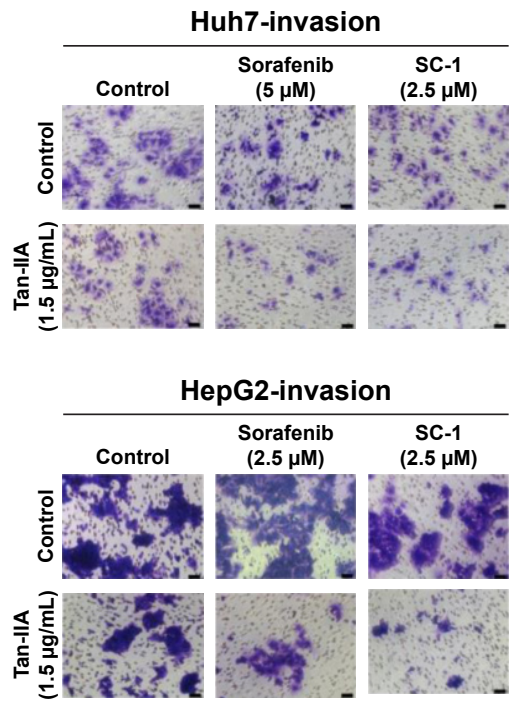

C

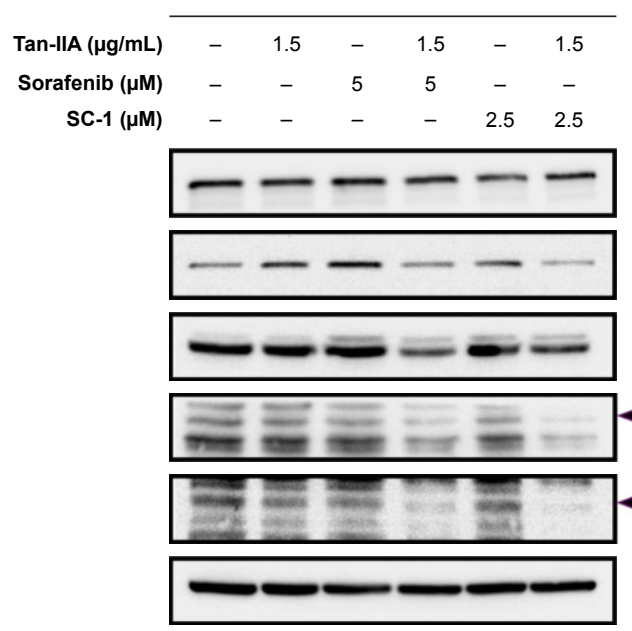

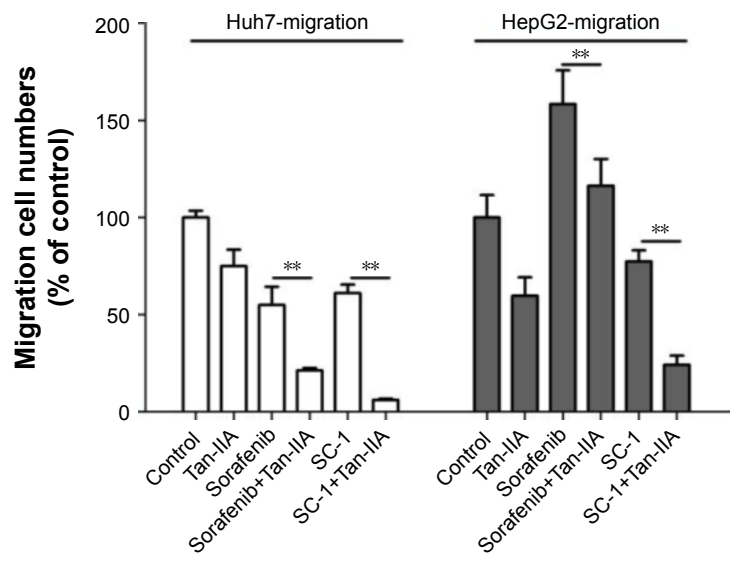
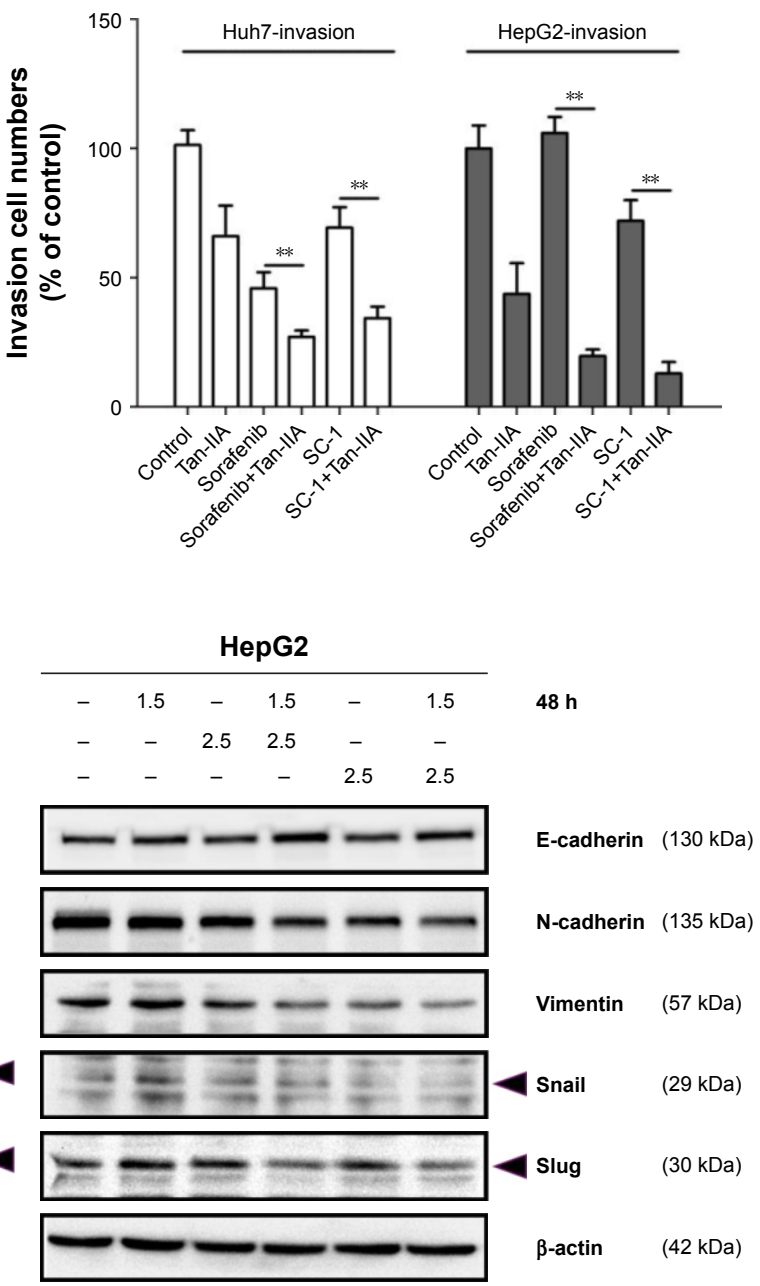

Figure 3 Effect of Tan-IIA combination treatment with sorafenib/SC-I in the migration and invasion on HCC cell lines.

Notes: Huh7 and HepG2 cells were treated with or without $1.5 \mu \mathrm{g} / \mathrm{mL}$ Tan-IIA in the presence or absence of 2.5 or $5 \mu \mathrm{M}$ sorafenib or $5 \mu \mathrm{M} \mathrm{SC-I} \mathrm{for} 24 \mathrm{~h}(\mathbf{A}$, migration assay) or $48 \mathrm{~h}$ (B, invasion assay) (C, Western blot analysis). Data are presented as mean \pm SD. $* * p<0.01$ versus sorafenib/SC-I alone groups.

Abbreviations: HCC, hepatocellular carcinoma; Tan-IIA, tanshinone IIA. 
A

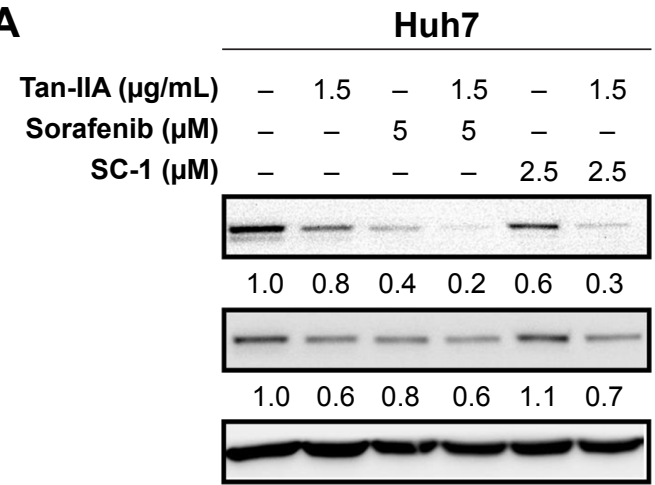

B

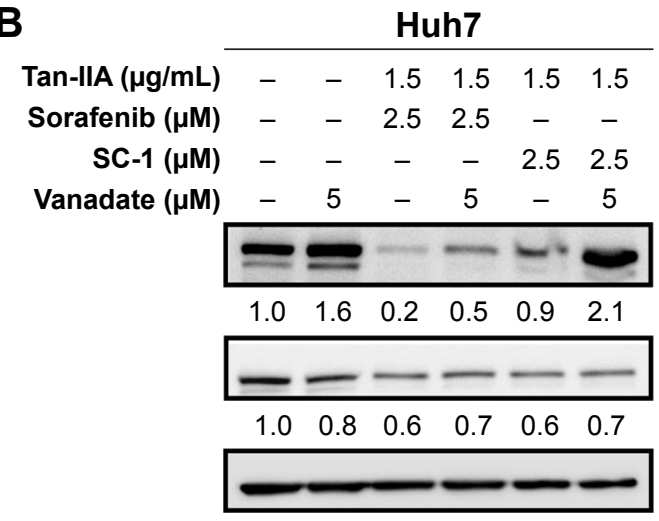

C

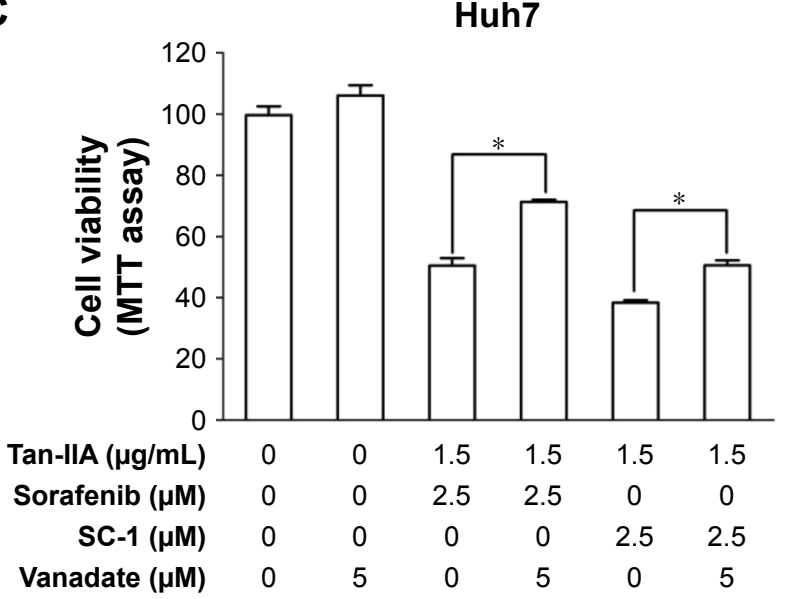

HepG2

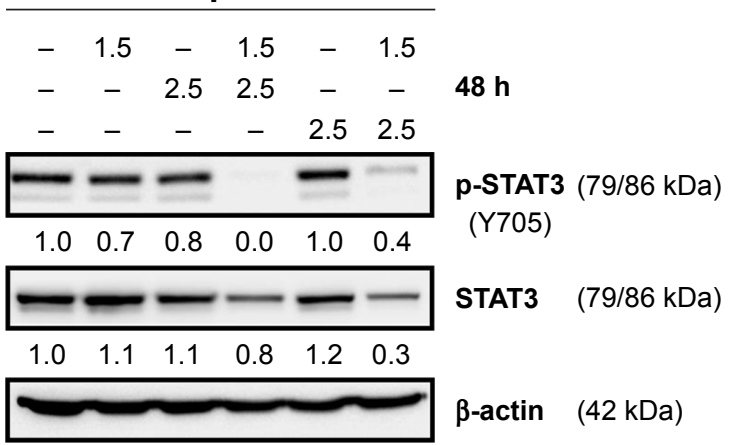

HepG2

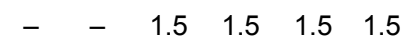

- $\quad 2.52 .5-\quad-48 \mathrm{~h}$

$-\quad-\quad-\quad-2.52 .5$

$-5-5-5$

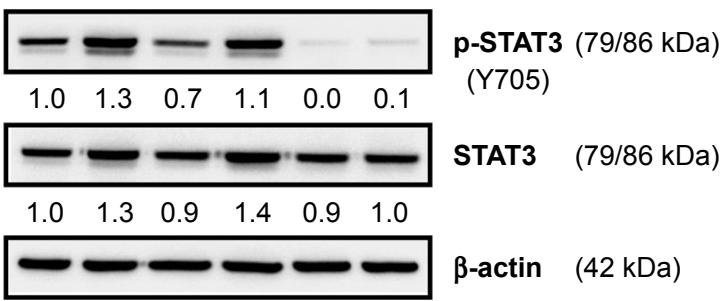

HepG2

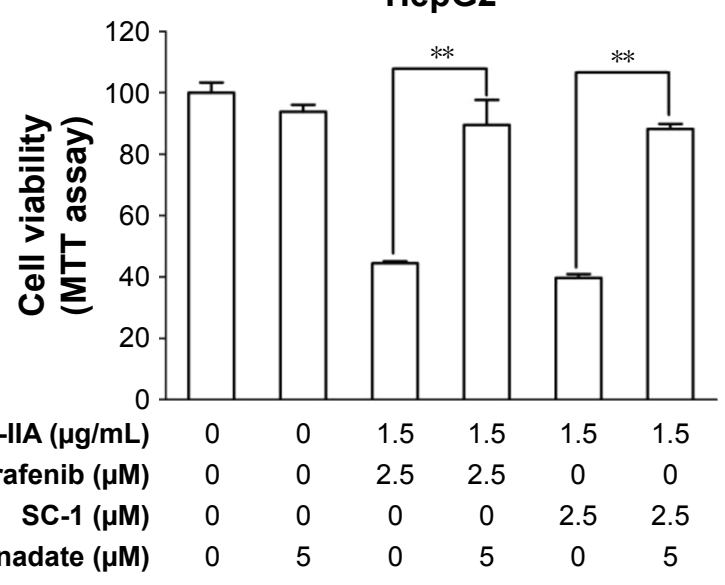

Figure 4 Effect of Tan-IIA combination treatments with sorafenib or SC-I on STAT3 signaling in HCC cell lines.

Notes: (A) Huh7 and HepG2 cells were treated with $1.5 \mu \mathrm{g} / \mathrm{mL}$ Tan-IIA in the presence or absence of 2.5 or $5 \mu \mathrm{M}$ sorafenib or $5 \mu \mathrm{M}$ SC-I for $48 \mathrm{~h}$ and assessed by Western blot analysis. (B) Huh7 and HepG2 cells were treated with $2.5 \mu \mathrm{M}$ sorafenib/SC-I combined with $1.5 \mu \mathrm{g} / \mathrm{mL}$ Tan-IIA in the presence or absence of sodium vanadate (a broadspectrum phosphatase inhibitor) for $48 \mathrm{~h}$ followed by Western blot and MTT assay analysis (C). Data are presented as mean \pm SD. ${ }^{*} p<0.05$, and $* * p<0.0 \mathrm{l}$ versus sorafenib/ SC-I combined with Tan-IIA.

Abbreviations: HCC, hepatocellular carcinoma; Tan-IIA, tanshinone IIA.

sorafenib/SC-1 efficacy and could prove to be an effective therapeutic strategy against HCC progression.

\section{Acknowledgment}

This work was supported by grants from the Taichung Tzu Chi Hospital, Buddhist Tzu Chi Medical Foundation, Taichung, Taiwan (TTCRD105-17, TTCRD105-20, TTCRD106-05). The funders had no role in study design, data collection and analysis, decision to publish, or preparation of the manuscript.

\section{Author contributions}

All authors contributed toward data analysis, drafting, critical revision of the paper, approval of the final version manuscript for publications, and agreed to be accountable for all aspects of the work. 


\section{Disclosure}

The authors report no conflicts of interest in this work.

\section{References}

1. Torre LA, Bray F, Siegel RL, Ferlay J, Lortet-Tieulent J, Jemal A. Global cancer statistics, 2012. CA Cancer J Clin. 2015;65(2):87-108.

2. Centers for Disease Control and Prevention (CDC). Hepatocellular carcinoma - United States, 2001-2006. MMWR Morb Mortal Wkly Rep. 2010;59(17):517-520.

3. Bruix J, Raoul JL, Sherman M, et al. Efficacy and safety of sorafenib in patients with advanced hepatocellular carcinoma: subanalyses of a phase III trial. J Hepatol. 2012;57(4):821-829.

4. Cheng AL, Kang YK, Chen Z, et al. Efficacy and safety of sorafenib in patients in the Asia-Pacific region with advanced hepatocellular carcinoma: a phase III randomised, double-blind, placebo-controlled trial. Lancet Oncol. 2009;10(1):25-34.

5. Chen KF, Tai WT, Huang JW, et al. Sorafenib derivatives induce apoptosis through inhibition of STAT3 independent of Raf. Eur J Med Chem. 2011;46(7):2845-2851.

6. Liu CY, Tseng LM, Su JC, et al. Novel sorafenib analogues induce apoptosis through SHP-1 dependent STAT3 inactivation in human breast cancer cells. Breast Cancer Res. 2013;15(4):R63.

7. Chen KF, Tai WT, Hsu CY, et al. Blockade of STAT3 activation by sorafenib derivatives through enhancing SHP-1 phosphatase activity. Eur J Med Chem. 2012;55:220-227.

8. Morimoto M, Numata K, Kondo M, et al. Field practice study of half-dose sorafenib treatment on safety and efficacy for hepatocellular carcinoma: a propensity score analysis. Hepatol Res. 2015;45(3): 279-287.

9. Chiu SC, Huang SY, Chen SP, Su CC, Chiu TL, Pang CY. Tanshinone IIA inhibits human prostate cancer cells growth by induction of endoplasmic reticulum stress in vitro and in vivo. Prostate Cancer Prostatic Dis. 2013;16(4):315-322.

10. Huang ST, Huang CC, Huang WL, et al. Tanshinone IIA induces intrinsic apoptosis in osteosarcoma cells both in vivo and in vitro associated with mitochondrial dysfunction. Sci Rep. 2017;7:40382.

11. Su CC, Chiu TL. Tanshinone IIA decreases the protein expression of EGFR, and IGFR blocking the PI3K/Akt/mTOR pathway in gastric carcinoma AGS cells both in vitro and in vivo. Oncol Rep. 2016;36(2): 1173-1179.

12. Wang JF, Feng JG, Han J, Zhang BB, Mao WM. The molecular mechanisms of Tanshinone IIA on the apoptosis and arrest of human esophageal carcinoma cells. Biomed Res Int. 2014;2014: 582730 .

13. Xie J, Liu J, Liu H, et al. The antitumor effect of tanshinone IIA on antiproliferation and decreasing VEGF/VEGFR2 expression on the human non-small cell lung cancer A549 cell line. Acta Pharm Sin B. 2015; 5(6):554-563.
14. Liu SP, Harn HJ, Chien YJ, et al. n-Butylidenephthalide (BP) maintains stem cell pluripotency by activating Jak2/Stat 3 pathway and increases the efficiency of iPS cells generation. PLoS One. 2012;7(9):e44024.

15. Cheng CY, Su CC. Tanshinone IIA inhibits Hep-J5 cells by increasing calreticulin, caspase 12 and GADD153 protein expression. Int $J$ Mol Med. 2010;26(3):379-385.

16. Lee WY, Chiu LC, Yeung JH. Cytotoxicity of major tanshinones isolated from Danshen (Salvia miltiorrhiza) on HepG2 cells in relation to glutathione perturbation. Food Chem Toxicol. 2008;46(1):328-338.

17. Yu H, Kortylewski M, Pardoll D. Crosstalk between cancer and immune cells: role of STAT3 in the tumour microenvironment. Nat Rev Immunol. 2007;7(1):41-51.

18. Kortylewski M, Yu H. Stat3 as a potential target for cancer immunotherapy. J Immunother. 2007;30(2):131-139.

19. Tai WT, Cheng AL, Shiau CW, et al. Dovitinib induces apoptosis and overcomes sorafenib resistance in hepatocellular carcinoma through SHP-1-mediated inhibition of STAT3. Mol Cancer Ther. 2012;11(2): 452-463.

20. Chen KF, Tai WT, Liu TH, et al. Sorafenib overcomes TRAIL resistance of hepatocellular carcinoma cells through the inhibition of STAT3. Clin Cancer Res. 2010;16(21):5189-5199.

21. Huang SY, Chang SF, Liao KF, Chiu SC. Tanshinone IIA inhibits epithelial-mesenchymal transition in bladder cancer cells via modulation of STAT3-CCL2 signaling. Int J Mol Sci. 2017;18(8):pii: E1616.

22. Chou TC. Drug combination studies and their synergy quantification using the Chou-Talalay method. Cancer Res. 2010;70(2):440-446.

23. Su JC, Tseng PH, Wu SH, et al. SC-2001 overcomes STAT3-mediated sorafenib resistance through RFX-1/SHP-1 activation in hepatocellular carcinoma. Neoplasia. 2014;16(7):595-605.

24. Chen W, Wu J, Shi H, et al. Hepatic stellate cell coculture enables sorafenib resistance in Huh7 cells through HGF/c-Met/Akt and Jak2/ Stat3 pathways. Biomed Res Int. 2014;2014:764981.

25. Xu J, Lin H, Li G, et al. Sorafenib with ASC-J9(R) synergistically suppresses the HCC progression via altering the pSTAT3-CCL2/Bcl2 signals. Int J Cancer. 2017;140(3):705-717.

26. Prete SD, Montella L, Caraglia M, et al. Sorafenib plus octreotide is an effective and safe treatment in advanced hepatocellular carcinoma: multicenter phase II So.LAR. study. Cancer Chemother Pharmacol. 2010; 66(5):837-844.

27. Hsu CH, Shen YC, Lin ZZ, et al. Phase II study of combining sorafenib with metronomic tegafur/uracil for advanced hepatocellular carcinoma. J Hepatol. 2010;53(1):126-131.

28. Chang TW, Lin CY, Tzeng YJ, Lur HS. Synergistic combinations of tanshinone IIA and trans-resveratrol toward cisplatin-comparable cytotoxicity in HepG2 human hepatocellular carcinoma cells. Anticancer Res. 2014;34(10):5473-5480.

29. Tai WT, Cheng AL, Shiau CW, et al. Signal transducer and activator of transcription 3 is a major kinase-independent target of sorafenib in hepatocellular carcinoma. J Hepatol. 2011;55(5):1041-1048.
OncoTargets and Therapy

\section{Publish your work in this journal}

OncoTargets and Therapy is an international, peer-reviewed, open access journal focusing on the pathological basis of all cancers, potential targets for therapy and treatment protocols employed to improve the management of cancer patients. The journal also focuses on the impact of management programs and new therapeutic agents and protocols on

\section{Dovepress}

patient perspectives such as quality of life, adherence and satisfaction. The manuscript management system is completely online and includes a very quick and fair peer-review system, which is all easy to use. Visit http://www.dovepress.com/testimonials.php to read real quotes from published authors. 